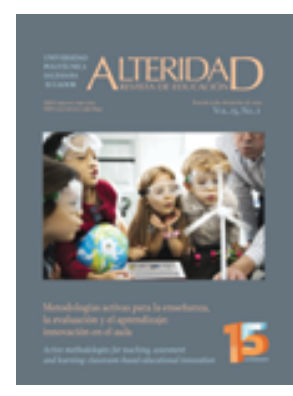

\title{
Revisión de propuestas metodológicas: Una taxonomía de agrupación categórica
}

\author{
Review of methodological proposals: \\ A categorical grouping taxonomy
}

Alejandro Rodríguez-García es docente e investigador de la Universidad de León (España) (arodrg01@estudiantes.unileon.es) (https://orcid.org/0000-0002-7258-8857)

Dra. Ana Rosa Arias-Gago es docente e investigadora de la Universidad de León (España) (ana.arias@unileon.es) (http://orcid.org/0000-0002-5889-3222)

Recibido: 2020-01-17 / Revisado: 2020-05-26 / Aceptado: 2020-06-05 / Publicado: 2020-07-01

\section{Resumen}

Actualmente, no existe ninguna taxonomía vinculada a la metodología que agrupe diferentes elementos metodológicos en función del carácter activo e instructivo de los mismos y de la etapa educativa a la que mejor se adecúen en términos de utilización. Por ello, el objetivo de esta investigación fue establecer una taxonomía considerando 76 recursos, estrategias, técnicas y métodos didácticos obtenidos tras una revisión de la principal literatura nacional e internacional. Para establecer la taxonomía, se elaboró y validó por medio del procedimiento de juicio de expertos la escala EVEMDT. La misma, fue administrada a un panel de 30 expertos quienes asistieron a un seminario de formación impartido por los investigadores, para valorar el carácter instructivo o activo y la adecuación a la etapa educativa de los 76 elementos metodológicos. Los resultados permitieron establecer una taxonomía donde aparecen 25 y 5 I recursos, estrategias, técnicas y métodos didácticos instructivos y activos respectivamente, clasificados también en función de la etapa educativa a la que mejor se adapten en términos de utilización. Se concluye que, las taxonomías con las que comparar los resultados son exiguas, aspecto que permite a esta ser un referente para los docentes a la hora de decidir qué recursos, estrategias, técnicas y métodos didácticos utilizar en función de la etapa educativa en la que se ubique el alumnado y el papel que quieran dotarle en sus procesos de aprendizaje.

Descriptores: Métodos de enseñanza, taxonomía, metodologías, educación activa, educación tradicional, enfoques educativos.

\section{Abstract}

Currently, there is no taxonomy linked to the methodology that groups different methodological elements based on both their active and instructive nature and the educational stage where they are best suited in terms of use. Hence, the objective of this research was to establish a taxonomy taking into account 76 resources, strategies, techniques and teaching methods obtained after a review of the main national and international literature. In order to establish the taxonomy, the EVEMDT scale was developed and validated through the expert judgment procedure. The EVEMDT scale was administered to a panel of 30 experts who attended a training seminar conducted by the researchers, to assess both the instructive or active nature and the 76 methodological elements adaptation to the educational stage. Results shows a taxonomy composed of 25 and 5 I didactic resources, didactic strategies, didactic techniques and didactic methods respectively, also classified according to the educational stage where they are best suited in terms of use. It was concluded that taxonomies to compare the results are reduced, so that this taxonomy could be a reference for teachers when deciding what resources, strategies, techniques and teaching methods to use depending both the students' educational stage and the role they want to give them in their learning processes.

Keywords: Teaching methods, taxonomy, methodologies, active education, traditional education, learning approaches.

Forma sugerida de citar: Rodríguez-García, A., \& Arias-Gago, A. (2020). Revisión de propuestas metodológicas: Una taxonomía de agrupación categórica. Alteridad, 15(2), 146-160. https://doi.org/10.17163/alt.v15n2.2020.01 


\section{Introducción y estado de la cuestión}

La metodología didáctica junto con la organización escolar y la evaluación educativa, son unos de los elementos más relevantes de la didáctica educativa y cobran un papel esencial en la praxis docente (Cantón \& Pino, 2014; FernándezBalboa, 2003; López-Pastor, 2009; Marina et al., 2015), sin embargo, a pesar de esta relevancia, es complejo encontrar una clasificación metodológica clara, exhaustiva y unificada.

Esta problemática surge porque cada autor elabora su listado de métodos didácticos en función de su experiencia y conocimiento sin tener en consideración los trabajos realizados por otros autores; también porque cada autor utiliza terminologías diferentes para referirse a métodos similares y porque existe una gran dispersión a la hora de enumerar diferentes métodos docentes (Alcoba, 2010, 2012).

Además, autores como Alcoba (2012), Palomares (2011) y Zemelman et al. (2005), establecen que la terminología vinculada a la metodología didáctica dispone de un carácter excesivamente críptico lo que genera que, términos como modelo, método, técnica, estrategia y recurso, sean utilizados, en muchos casos, como sinónimos. Este aspecto genera una gran confusión terminológica en la comunidad educativa, de esta forma, para tratar de clarificar esta circunstancia, en la Tabla 1, se ha establecido una definición de cada uno de los elementos, a los que se le ha asociado un ejemplo vinculado a la práctica educativa.

Tabla 1. Delimitación conceptual y ejemplificaciones

\begin{tabular}{|c|c|c|c|}
\hline Modelo didáctico & Método didáctico & $\begin{array}{l}\text { Estrategia/técnica } \\
\text { didáctica }\end{array}$ & Recursos didácticos \\
\hline $\begin{array}{l}\text { Componentes educativos } \\
\text { sustentados en una teoría } \\
\text { educativa que permiten de- } \\
\text { terminar los propósitos, mé- } \\
\text { todos y recursos a utilizar, } \\
\text { así como la organización } \\
\text { y evaluación a implemen- } \\
\text { tar durante el proceso de } \\
\text { enseñanza-aprendizaje } \\
\text { (Zemelman et al., 2005). }\end{array}$ & $\begin{array}{l}\text { Conjunto de actuaciones } \\
\text { que un docente utiliza con } \\
\text { el fin de alcanzar los obje- } \\
\text { tivos educativos, que tiene } \\
\text { sentido como un todo y que } \\
\text { responde a una denomina- } \\
\text { ción aceptada por la comu- } \\
\text { nidad científica } \\
\text { (Alcoba, 2012). }\end{array}$ & $\begin{array}{l}\text { Concreciones sustentadas } \\
\text { en un método didáctico, } \\
\text { organizadas y planificadas } \\
\text { por el docente, que tienen } \\
\text { por objeto la construcción } \\
\text { del aprendizaje a través de } \\
\text { actividades y tareas en las } \\
\text { que se integran. } \\
\text { (Alcoba, 2012). }\end{array}$ & $\begin{array}{l}\text { Conjunto de elementos tan- } \\
\text { gibles o intangibles, que } \\
\text { alumnado y/o docentes, uti- } \\
\text { lizan como soporte y/o com- } \\
\text { plemento en sus procesos } \\
\text { de enseñanza y aprendizaje } \\
\text { (Díaz-Lucea, 1996). }\end{array}$ \\
\hline Ejemplo & Ejemplo & Ejemplo & Ejemplo \\
\hline Modelo activo. & Aprendizaje cooperativo. & Folio giratorio. & $\begin{array}{l}\text { Folio, bolígrafo, } \\
\text { temporizador. }\end{array}$ \\
\hline
\end{tabular}

\section{Fuente: Elaboración propia}

Si a las problemáticas anteriores, le sumamos la "pirotecnia" de uso y creación de nuevos enfoques metodológicos como consecuencia del frenesí innovador de muchos docentes (PérezPueyo \& Hortigüela, 2020) en la era de las redes sociales, se genera el caldo de cultivo perfecto para que sea extremadamente complejo agrupar y clasificar los mismos. Por ello, en el presente estudio, se pretende establecer una taxonomía clasificatoria de los principales recursos didácticos, estrategias, técnicas y métodos didácticos en función de 3 criterios: (1) carácter activoinstructivo, (2) tipología (recurso didáctico, estrategia/técnica y/o método didáctico y (3) adecuación en términos de utilización en una o varias etapas educativas (Infantil y Primaria, 
Secundaria y Bachillerato, Educación Superior y todas las etapas educativas).

De esta forma, los métodos docentes con carácter activo, los cuales se vinculan al criterio 1, se definen como: "Aquellos métodos, técnicas y estrategias que utiliza el docente para convertir el proceso de enseñanza-aprendizaje en actividades que fomenten la participación activa del estudiante y su protagonismo" (Andreu-Andrés \& Labrador-Piquer, 2011, p. 6). De esta forma, la utilización de estas metodologías genera que el docente adopte el rol de facilitador o guía del aprendizaje del alumno. Este aspecto otorga al alumnado protagonismo, dotándolos de mayor motivación, participación, cooperación, autonomía y autorregulación (Tourón \& Santiago, 2015). Como contraposición a los métodos activos, aparecen los métodos docentes de corte tradicional, transmisivo o instructivo, los cuales, son definidos como: "Aquellos métodos, técnicas y estrategias que buscan el aprendizaje conceptual y procesual del alumno, a través de la instrucción del docente y de la recepción del alumnado" (Toro \& Arguis, 2015, p. 4).

Para establecer la taxonomía, se llevó a cabo una revisión bibliográfica en las bases de datos Scopus, Web of Science, Dialnet, ERIC y Education Data. La revisión permitió la obtención de los siguientes 76 recursos, estrategias, técnicas y métodos didácticos: Lección magistral, lección magistral participativa, autorregulación del aprendizaje, áreas o rincones de trabajo, asamblea, trabajo por proyectos, aprendizaje mediante simulación, aprendizaje basado en problemas, aprendizaje basado en pensamiento, comunidades de aprendizaje, Flipped classroom, aprendizaje mediante portfolio, centros de interés, método Reggio Emilia, método Montessori, método Waldorf, talleres, respuesta física total o total physical response (TPR), método Pikler, método Aucouturier, aprendizaje por descubrimiento, descubrimiento guiado, coaching educativo, bits de inteligencia, aprendizaje cooperativo, cuñas motrices o descansos activos, método de instrucción estratégica y autorregulada (SRSD), aprendizaje mediante grupos de discusión, estudio de casos, aprendizaje basado en indagación, aprendizaje por observación, mobile learning, metodología CLIL, estilo actitudinal, contratos didácticos, mindfulness, ciclo de Kolb, aprendizaje por organizadores gráficos, uso del método científico, grupos interactivos, cálculo abierto basado en número $(\mathrm{ABN})$, e-learning, gamificación, aprendizaje servicio, tutoría sujeto-sujeto o tutoría entre iguales, aprendizaje mediante discusión o debate, just-in-time teaching, método de preparación y estudio previo por evaluación on-line automática (PEPEOLA), sistema Amara Berri, método RULER para el desarrollo emocional, aprendizaje por pares, aprendizaje por telecolaboración o intercambios virtuales, método Singapur, aprendizaje significativo, método teacch, método phonics, programación neurolingüística (PNL), proyectos de comprensión inteligente, aprendizaje mediante realidad virtual o aumentada (VR y RA), aprendizaje mediante foros de discusión, modelado cognitivo, moldeado cognitivo, pedagogía sistémica, aprendizaje mediante copiado, aprendizaje mediante dictado, aprendizaje a través de lecturas de textos, aprendizaje mediante video-tutoriales, paisajes de aprendizaje, aprendizaje mediante chroma key, aprendizaje mediante videos y películas, aprendizaje mediante webquest, aprendizaje a través de redes sociales, aprendizaje basado en juegos, aprendizaje basado en retos, escape room y break out educativos y Kunskapsskolan (Andreu-Andrés \& Labrador-Piquer, 2011, Blackshields et al., 2016; Blanchard \& Muzás, 2016; Bourner, 1997; De Miguel, 2009; Educacyl, 2019; Hernández \& Guárate, 2017; Luelmo, 2018; Nieto \& Alfageme-González, 2017; Navaridas, 2004; Paños, 2017; Prieto et al., 2014; RodríguezGarcía \& Arias-Gago, 2019).

También fue necesario considerar las características que hacen que una metodología sea activa ya que estas, sirvieron, para establecer las dimensiones de evaluación de la escala que permitió llevar a cabo la taxonomía. Así, tomando en consideración a Borko et al. (2010), 
Crisol (2012), De Miguel (2009), FernándezMarch (2006), Gil (2014), Palma et al. (2017), Palomares (2011), Rodríguez-García y AriasGago (2019), Silva y Maturana (2017), Toro y Arguis (2015), Vallejo y Molina (2011) y Zabalza (2003), se podría sintetizar que las características que originan que un enfoque metodológico sea activo, son las siguientes: Debe partir de los intereses, necesidades y motivaciones del alumnado; el alumnado debe de aprender haciendo y con la práctica en situaciones contextualizadas; tiene que generar motivación intrínseca hacia el aprendizaje; debe promover la creatividad, la crítica y el sentido de iniciativa y espíritu emprendedor; debe desarrollar las relaciones interpersonales y la inserción social del alumnado por medio del trabajo cooperativo y colaborativo; debe de asociarse a una evaluación comprensiva y auténtica con las características del alumnado; debe de ser un medio para que el alumnado alcance la autonomía intelectual y moral; se tiene que fundamentar en tópicos generadores y globalizados adaptados a los intereses del alumnado; necesita disponer de una organización de los espacios, agrupamientos y tiempos flexible; debe sustentarse en la colaboración y cooperación del alumnado a través de la creación de grupos heterogéneos; debe de valerse de las TIC para generar aprendizajes integrados y motivantes para el alumnado; el docente tiene que actuar como guía y facilitador del aprendizaje; tiene que implicar a todos los miembros de la comunidad educativa (familia, alumnado, docentes e instituciones); se tiene que implementar con actividades y tareas ubicadas en la zona de desarrollo próximo del alumnado; debe atender a la diversidad del alumnado, permitiendo una enseñanza individualizada e inclusiva con todo el alumnado; y debe de propiciar el aprendizaje lógico, el desarrollo del pensamiento hipotético deductivo, la resolución de problemas en situaciones contextualizadas y el pensamiento crítico.

Por tanto, considerando los recursos, estrategias, técnicas y métodos, las características expuestas y las etapas educativas, se creó una escala de valoración que fue administrada a una serie de expertos con el objetivo de establecer una taxonomía en base a criterios objetivos definiendo los recursos, estrategias, técnicas y métodos didácticos en función del carácter activo e instructivo y en función de la adecuación en términos de utilización a una o varias etapas educativas.

\section{Metodología}

\subsection{Diseño de investigación}

Se ha utilizado un diseño de investigación mixto cualitativo-cuantitativo en el que, en un primer momento, se utilizó el método de la revisión integradora. La misma, según Guirao (2015), se caracteriza en establecer una síntesis sobre conocimientos teóricos, metodológicos o investigaciones realizadas, en aras de esbozar una construcción teórica y/o conclusión sobre un tema específico vinculado. La revisión se llevó a cabo en las bases de datos multidisciplinares Scopus, Web of Science y Dialnet focalizando la búsqueda hacia áreas educativas. Por su parte, también se utilizaron las bases de datos ERIC y Education Data Base, las cuales, se vinculan específicamente al campo educativo. Se seleccionaron estas bases de datos por integrar repositorios, librerías electrónicas y revistas de alto impacto a nivel nacional e internacional. También, por estar disponibles en el catálogo de bases de datos de la Universidad de León (España) (afiliación de los autores).

La revisión realizada permitió delimitar 124 fuentes bibliográficas que, tras un proceso de revisión y análisis, dieron como resultado la obtención de 76 recursos, estrategias, técnicas y métodos didácticos. El principal criterio de inclusión en el listado, radicó en que cada propuesta o elemento metodológico fuese citado, al menos, en 3 fuentes bibliográficas diferentes.

Posteriormente, utilizando la información obtenida con la revisión integradora se elaboró la escala para la valoración de los enfoques metodológicos docentes transversales (EVEMDT), en 
la que se han plasmado los 76 recursos, estrategias, técnicas y métodos didácticos.

Tras la elaboración y validación de esta escala, se pasó a la parte del diseño de investigación cuantitativa ex post-facto (Colás et al., 2009), donde se administró la escala a un panel de expertos en servicio activo docente con amplios conocimientos en la temática de métodos docentes para, de esta forma, establecer la taxonomía sobre metodología didáctica.

\subsection{Participantes}

La muestra estuvo compuesta por un panel de $30(n=30)$ profesores expertos en metodologías docentes a los que se les administró la escala EVEMDT. De estos 30 participantes, $22(n=22)$ eran maestros en activo que estaban cursando el Máster de investigación en Psicología y Ciencias de la Educación en la Universidad de León y que asistieron en una de las asignaturas del citado Máster a un seminario teórico-práctico de 20 horas donde los investigadores les instruyeron sobre los 76 recursos, estrategias, técnicas y métodos didácticos y, además, elaboraron un trabajo teórico-práctico sobre los mismos.

Otros componentes del panel de expertos fueron doctores en Educación y profesores-doctores de la Universidad de León con un amplio conocimiento sobre la materia y a los que se les instruyó sobre los enfoques no conocidos $(n=4)$; también orientadores educativos egresados en pedagogía, que actualmente prestan servicio en la Comunidad Autónoma de Castilla y León $(n=2)$, a los que también se les instruyó sobre los enfoques no conocidos y; finalmente, los propios investigadores del estudio $(n=2)$, quienes también efectuaron la valoración utilizando la escala y se encargaron de instruir al panel de expertos.

El hecho de estar en servicio activo docente, junto con disponer o estar cursando los estudios de Máster y/o doctorado en el campo de las Ciencias de la Educación, así como la asistencia al seminario teórico-práctico sobre métodos docentes, son los criterios que se han tenido en cuenta para considerar a los participantes como expertos en la materia.

\subsection{Instrumento}

Para establecer la taxonomía se diseñó y elaboró ad-hoc la escala EVEDMT para valorar y clasificar los 76 recursos, estrategias, técnicas y métodos didácticos seleccionados. Para la elaboración y diseño de la escala se siguió el siguiente procedimiento:

Análisis previo de la literatura: Se implementó una revisión integradora con la que seleccionaron los 76 elementos citados. Esta revisión también sirvió para configurar las dimensiones de valoración de la escala.

Elaboración de la escala EVEDMT: Se diseñó la escala por medio de la especificación, redacción y ordenación de las 20 dimensiones de la escala a las que se le asociaron los 76 enfoques metodológicos (Tabla 2). Cada dimensión en cada enfoque metodológico, fue valorada con una escala de 0 a 4 puntos, donde 0 se corresponde con la no tenencia de una característica y 4 con la máxima tenencia. A su vez, cada recurso, estrategia, técnica y método fue asociado a la etapa o etapas educativas con las que se adecúan en términos de utilización. Para este propósito, cada participante asoció cada elemento a una o varias etapas. En este sentido, las categorías vinculadas a las etapas educativas fueron: 1 . Educación Infantil y Primaria, 2. Educación Secundaria y Bachillerato, 3. Educación Superior, 4. Todas las etapas educativas.

Validación de la escala: Se utilizó el procedimiento del juicio de expertos. Para la validación de contenido, intervinieron 5 expertos, de los cuales, 2 fueron profesores-doctores de la Universidad de León y expertos en la materia; otros 2 fueron orientadores educativos en activo en la Comunidad Autónoma de Castilla y León; y otro asesor del Centro de Formación del profesorado e innovación educativa de la ciudad de León. 


\section{Tabla 2. Indicadores utilizados para la elaboración de la taxonomía}

\section{El enfoque metodológico...}

1. Parte de intereses del alumnado.

2. Genera aprendizaje contextualizado.

3. Genera motivación intrínseca.

4. Desarrolla creatividad, pensamiento crítico y emprendimiento del alumno.

5. Desarrolla socialmente al alumno.

6. Propicia evaluación auténtica y comprensiva.

7. Desarrolla la autonomía y la autorregulación.

8. Parte de tópicos generadores y globalizados.

9. Requiere una organización de espacios, tiempos y agrupamientos flexible.

10. Requiere trabajo colaborativo.

11. Requiere utilización de las TIC.

12. Docente actúa como guía y facilitador de aprendizaje.

13. Implica a toda la comunidad educativa.

14. Requiere ejercicios, actividades, tareas, problemas y proyectos que se ubiquen en la Zona de Desarrollo Próximo del alumnado.

15. Atiende a la diversidad del alumnado y propicia la individualización de la enseñanza.

16. Propicia la inclusión del alumnado.

17. Desarrolla el pensamiento hipotético-deductivo y la abstracción.

18. Desarrolla competencialmente al alumno.

19. Desarrolla la comunicación y el lenguaje del alumno.

20. Parte de situaciones problemas del mundo real que fomentan la práctica de situaciones integradas

\section{Fuente: Elaboración propia}

Determinación de la fiabilidad del cuestionario: Se utilizó el procedimiento Alfa de Cronbach. La fiabilidad de la escala fue alta con un valor de .85 , obteniendo para los ítems un $\mathrm{r}=.856$, el cual, sobrepasa según Castañeda et al. (2010), el límite inferior considerado como fiable.

Redacción final de la escala EVEDMT: La escala quedó finalmente compuesta por 20 dimensiones a valorar en cada uno de los 76 enfoques metodológicos seleccionados como consecuencia de aparecer en, al menos, 3 fuentes de la revisión. Además, cada elemento tuvo que ser integrado en la etapa o etapas educativas a la que mejor se adecúe en términos de utilización.

Para la valoración de los elementos metodológicos, una vez cumplimentada la escala por cada uno de los participantes, se diseñó un sistema de categorías exhaustivo y mutuamente excluyente (Tabla 3), con el que valorar el carácter activo o instructivo de cada enfoque metodológico.

Tabla 3. Sistema de categorías exhaustivo y mutuamente excluyente

\begin{tabular}{|c|c|}
\hline Categoría & Puntuaciones \\
\hline Recursos, estrategias, técnicas y métodos instructivos & $0-2$ \\
\hline Recursos, estrategias, técnicas y métodos activos & $2,0001-4$ \\
\hline
\end{tabular}

Fuente: Elaboración propia 
Por su parte, para asociar a cada recurso, estrategia, técnica y método a una etapa educativa concreta, se estableció que, al menos, debería de haber una frecuencia mínima de 10 selecciones, de esta forma, se minimiza el sesgo que se puede ocasionar cuando un elemento metodológico genera dudas en los participantes a la hora de su clasificación.

\subsection{Análisis estadísticos}

Se realizó con la versión 26 del programa SPSS, considerando los criterios de Tejedor y GarcíaValcarcel (2012) e implementando los siguientes análisis:

Análisis de valores promedio y de frecuencias: Los valores promedio se utilizaron para establecer el carácter activo o instructivo de los 76 elementos metodológicos integrados en la escala en función del sistema de categorías establecido. A su vez, las frecuencias se utilizaron para clasificar los 76 elementos metodológicos en una o varias etapas educativas.

\section{Resultados}

\subsection{Recursos didácticos, estrategias, técnicas y métodos instructivos}

La clasificación resultante tras la valoración realizada por el panel de expertos seleccionado, aparece reflejada en la Tabla 4. En la misma, se muestran de mayor a menor carácter instructivo, los diferentes recursos, estrategias, técnicas y métodos seleccionados en la revisión realizada. A este respecto, el número de elementos metodológicos instructivos asciende a 25 , número significativamente inferior al de los elementos activos que, se compone, de 51. En la clasificación, también se ha valorado la tipología a la que pertenecen (método, técnica y/o estrategia y recursos didácticos) y la etapa educativa a la que mejor se adecúan en términos de utilización.
De esta forma, en las etapas de Educación Infantil y Primaria, los métodos instructivos resultantes ordenados en función del mayor carácter instructivo, son los siguientes: Total Physical response $\bar{x}=1.2$, Phonics $\bar{x}=1.25$, Método Teacch $\bar{x}=1.45$, método de instrucción estratégica $y$ autorregulada para el aprendizaje de la escritura (SRSD) $\bar{x}=1.6$ y método CLIL $\bar{x}=1.65$. Por su parte, la estrategia/técnica que aparece en las etapas de Educación Infantil y Primaria, es los bits de inteligencia $\bar{x}=.85$.

En las etapas de Secundaria y Bachillerato, los métodos que aparecen son los siguientes: Phonics $\bar{x}=1.25$, coaching educativo $\bar{x}=1.5$ y método CLIL $\bar{x}=1.65$. Tanto el coaching educativo como el método CLIL, se adecúan también a la Educación Superior y a las etapas de Secundaria y Bachillerato respectivamente.

A nivel de Educación Superior o Universitario, el único método que se ha clasificado como instructivo es el coaching educativo $\bar{x}=1.5$, cuya utilización también se adecúa a las etapas de Secundaria y Bachillerato.

Por su parte, aparecen diversos métodos, técnicas/estrategias y recursos didácticos que se adecúan en términos de utilización a todas las etapas mencionadas. De esta manera, los métodos resultantes, son los siguientes: Lección magistral $\bar{x}=.5$, lección magistral participativa $\bar{x}=.6$, aprendizaje por observación $\bar{x}=1$, Mindfulness $\bar{x}=1.45$, Ciclo de Kolb $=1.75 y$ e-learning $\bar{x}=1.8$. A su vez, las estrategias/técnicas integradas en la taxonomía son las siguientes: Aprendizaje mediante copiado $\bar{x}=.4$, aprendizaje mediante dictado $\bar{x}=.45$, contratos didácticos $\bar{x}=.7$, modelado $\bar{x}=.75$, moldeado $\bar{x}=.8$, aprendizaje mediante lecturas $\bar{x}=.9$, aprendizaje mediante video-tutoriales $\bar{x}=1.35$, grupos de discusión $\bar{x}=1.65$ y paisajes de aprendizaje $\bar{x}=1.95$. Finalmente, recursos didácticos como los organizadores gráficos $\bar{x}=1.35$, el portfolio $\bar{x}=1.4$ y los foros de discusión $\bar{x}=1.9$, se adecúan también a todas las etapas educativas. 


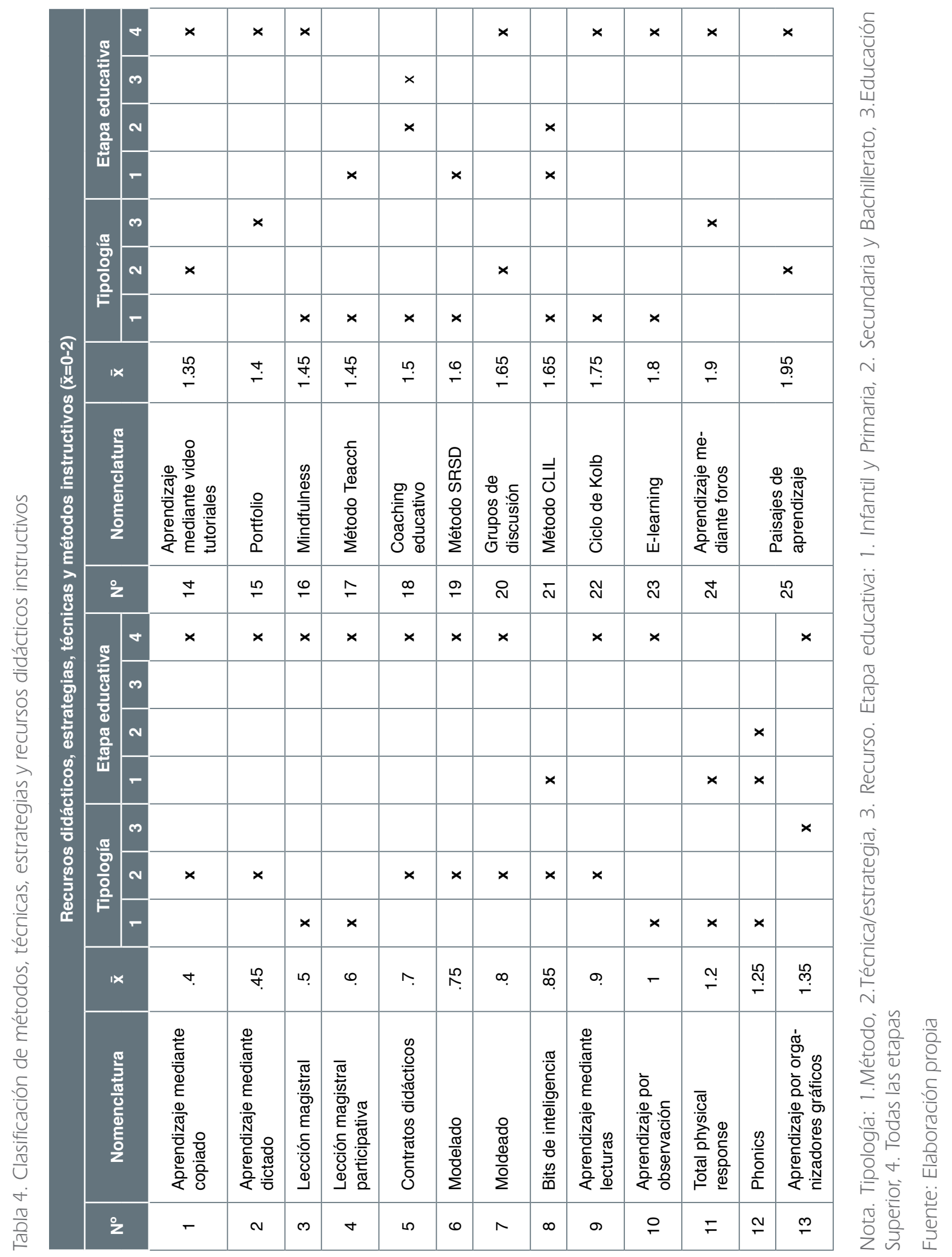




\subsection{Recursos didácticos, estrategias, técnicas y métodos activos}

Como en el apartado anterior, la Tabla 5 explicita la clasificación realizada por el panel de expertos y, en la misma, se ubican de mayor a menor carácter activo, diferentes recursos, estrategias/ técnicas y métodos seleccionados en la revisión realizada. En la presente clasificación, el número de elementos incluidos es significativamente mayor que en el caso anterior (51 a 25).

Continuando con la agrupación realizada, en las etapas de Educación Infantil y Primaria, se han incluido en función de mayor a menor carácter activo, los siguientes métodos: Amara Berri $\bar{x}=3.7$, Kunskapsskolan $\bar{x}=3.65$, método Montessori $\bar{x}=3.6$, Reggio Emilia $x=3.4$, comunidades de aprendizaje $\bar{x}=3.2$, método Aucouturier $\bar{x}=3.15$, método Pikler $\bar{x}=3.15$, proyectos de comprensión inteligente $\bar{x}=3.05$, cálculo abierto basado en Número $(A B N) \bar{x}=2.95$, método Singapur $\bar{x}=2.9$, método Waldorf $\bar{x}=2.85$, descubrimiento guiado $\bar{x}=2.8$, rincones $\bar{x}=2.75$, método Ruler $x=2.6$, asamblea $\bar{x}=2.5$, pedagogía sistémica $\bar{x}=2.4$ $y$ programación neurolingüística (PNL) $\bar{x}=2.35$.

En las etapas de Secundaria y Bachillerato, los métodos incluidos son los siguientes: Comunidades de aprendizaje $\bar{x}=3.2$, proyectos de comprensión inteligente $\bar{x}=3.05$, mobile learning $\bar{x}=2.7$, pedagogía sistémica $x=2.4$, just in time teaching $\bar{x}=2.4$, método de preparación y estudio previo por evaluación on-line automática (PEPEOLA) $\bar{x}=2.15$ y aprendizaje por telecolaboración $\bar{x}=2.05$. A su vez, las estrategias/técnicas integradas en la taxonomía fueron: Aprendizaje mediante redes sociales $\bar{x}=2.68$ y tutoría entre iguales $\bar{x}=2.1$.

En la Educación Superior o ámbito universitario, los métodos incluidos, coincidentes con los de las etapas de Secundaria y Bachillerato, son los siguientes: Mobile learning $\bar{x}=2.7$, just in time teaching $\bar{x}=2.4$, método de preparación y estudio previo por evaluación on-line automática (PEPEOLA) $\bar{x}=2.15$ y aprendizaje por telecolaboración $\bar{x}=2.05$. Por su parte, las estrategias/ integradas son iguales a los 2 de las etapas de Secundaria y Bachillerato.

En último término y como en el caso anterior, existen diversos métodos, estrategias, técnicas y recursos didácticos que se adecúan $y$ pueden ser utilizados en todas las etapas educativas mencionadas. De esta forma, los métodos integrados en esta categoría son los siguientes: Trabajo por proyectos $\bar{x}=3.7$, aprendizaje cooperativo 3.35 , aprendizaje basado en problemas $\bar{x}=3.3$, aprendizaje basado en retos $\bar{x}=3.25$, aprendizaje servicio $\bar{x}=3.25$, gamificación $\bar{x}=3.25$, estilo actitudinal $\bar{x}=3.2$, estudio de casos $\bar{x}=3.1$, aprendizaje basado en juegos $\bar{x}=3$, centros de interés 2.9 , aprendizaje por pares $\bar{x}=2.85$, autorregulación del aprendizaje $\bar{x}=2.82$, aprendizaje por descubrimiento $\bar{x}=2.8$, talleres $\bar{x}=2.78$, Flipped classroom $\bar{x}=2.75$, aprendizaje por indagación $\bar{x}=2.65$, aprendizaje significativo $\bar{x}=2.55$ y aprendizaje basado en pensamiento $\bar{x}=2.55$. A su vez, las estrategias/técnicas integradas en la taxonomía en función de su adecuación y utilización en todas las etapas educativas, son las siguientes: Escape room y break out educativo $\bar{x}=3.35$, grupos interactivos $\bar{x}=3.1$, aprendizaje mediante realidad virtual y realidad aumentada (VR y RA) $\bar{x}=3.05$, uso del método científico $x=2.7$, simulación $\bar{x}=2.65$, aprendizaje mediante debate $\bar{x}=2.6$ y cuñas motrices o descansos acti$\operatorname{vos} \bar{x}=2.1$. Finalmente, recursos didácticos como la webquest $\bar{x}=2.65$, el aprendizaje mediante videos y películas $\bar{x}=2.45$ y el aprendizaje mediante chroma Key $\bar{x}=2.25$, se adecúan y se utilizan en todas las etapas educativas. 


\begin{tabular}{|c|c|c|c|c|c|c|c|c|c|c|c|c|c|c|c|c|c|c|}
\hline & $\Phi$ & $\theta$ & $x$ & & $x$ & $x$ & & & $x$ & & $x$ & $x$ & $x$ & & $x$ & $x$ & $x$ & \\
\hline & ల్ల & $\infty$ & & & & & & $x$ & & $x$ & & & & & & & & \\
\hline & $\underset{\mathbb{N}}{\mathbb{\infty}}$ & N & & & & & & $x$ & & $x$ & & & & & & & & \\
\hline & 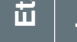 & - & & $x$ & & & $x$ & & & & & & & $x$ & & & & $x$ \\
\hline & $\pi$ & $\infty$ & & & & & & & & & & & $x$ & & & & & \\
\hline & 응 & N & & & & & & & $x$ & $x$ & & $x$ & & & $x$ & & & \\
\hline & & - & $x$ & $x$ & $x$ & $x$ & $x$ & $x$ & & & $x$ & & & $x$ & & $x$ & $x$ & $x$ \\
\hline$f$ & $1 x$ & & $\stackrel{\infty}{\stackrel{N}{N}}$ & $\stackrel{\infty}{\sim}$ & $\stackrel{\infty}{\stackrel{\infty}{\alpha}}$ & $\stackrel{\stackrel{R}{N}}{\text { N }}$ & $\stackrel{\stackrel{R}{\sim}}{\text { N }}$ & $\hat{\mathrm{N}}$ & $\hat{i}$ & $\begin{array}{l}\stackrel{\infty}{\leftrightarrow} \\
\stackrel{\mathrm{N}}{*}\end{array}$ & 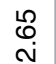 & \begin{tabular}{l}
$\ell$ \\
\hdashline \\
\end{tabular} & 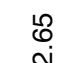 & $\stackrel{\circ}{\mathrm{N}}$ & $\stackrel{\circ}{\mathrm{N}}$ & 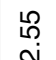 & مِّم & $\stackrel{\leftrightarrow}{\sim}$ \\
\hline 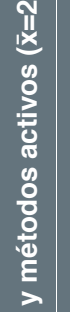 & 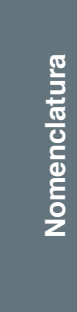 & & 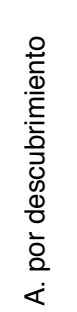 & 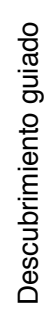 & 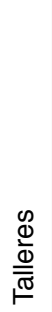 & 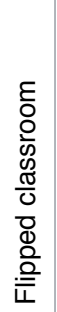 & 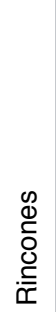 & 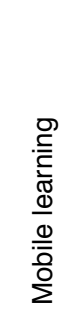 & 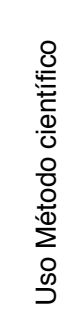 & $\begin{array}{l}0 \\
\frac{0}{0} \\
\frac{0}{0} \\
0 \\
0 \\
0 \\
0 \\
\frac{0}{0} \\
\frac{0}{2} \\
\overline{0} \\
0 \\
\dot{<}\end{array}$ & 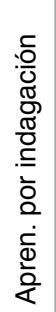 & 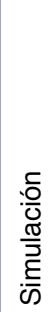 & 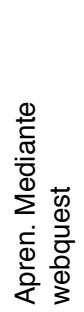 & 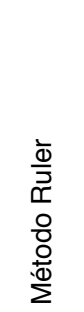 & 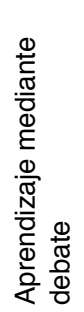 & 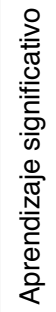 & 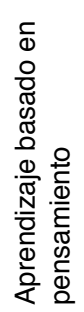 & 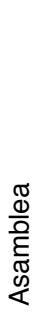 \\
\hline$\frac{\mathrm{O}}{\mathrm{C}}$ & $\stackrel{\circ}{z}$ & & $\hat{N}$ & $\stackrel{\infty}{N}$ & N & ஜ & m & $\tilde{ల}$ & ల్ల & से & $\stackrel{m}{m}$ & $\stackrel{\infty}{\infty}$ & 网 & $\stackrel{\infty}{\infty}$ & প্ল & 우 & $\bar{\gamma}$ & ₹ \\
\hline 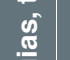 & 『 & $\theta$ & $x$ & & & & & $x$ & $x$ & $x$ & $x$ & $x$ & $x$ & & $x$ & & & $x$ \\
\hline 迎 & 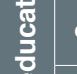 & $\infty$ & & & & & & & & & & & & & & & & \\
\hline $\begin{array}{c}0 \\
0 \\
0\end{array}$ & $\approx$ & $N$ & & & & & & & & & & & & $x$ & & & & \\
\hline$\frac{\mathrm{J}}{\mathrm{c}}$ & 岌 & - & & $x$ & $x$ & $x$ & $x$ & & & & & & & $x$ & & $x$ & $x$ & \\
\hline $\begin{array}{l}\text { 응 } \\
08\end{array}$ & $\stackrel{\Xi}{\cong}$ & $\infty$ & & & & & & & & & & & & & & & & \\
\hline 号 & $\div$ & $\mathbf{N}$ & & & & & & $x$ & & & & & & & & & & $x$ \\
\hline & & $\tau$ & $x$ & $x$ & $x$ & $x$ & $x$ & & $x$ & $x$ & $x$ & $x$ & $x$ & $x$ & $x$ & $x$ & $x$ & \\
\hline & $\mid x$ & & ஸे & $\hat{\infty}$ & $\begin{array}{l}\stackrel{\leftrightarrow}{\oplus} \\
\stackrel{\mu}{\infty}\end{array}$ & $\stackrel{0}{\oplus}$ & $\stackrel{\oplus}{\oplus}$ & 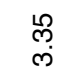 & 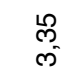 & $\stackrel{m}{m}$ & $\stackrel{\stackrel{\sim}{N}}{\underset{m}{\infty}}$ & $\begin{array}{l}\stackrel{n}{n} \\
\text { ஸ口 }\end{array}$ & $\underset{\sim}{\stackrel{\sim}{N}}$ & $\stackrel{\sim}{\sim}$ & $\underset{\sim}{N}$ & $\frac{L}{\infty}$ & $\frac{n}{\infty}$ & $\bar{m}$ \\
\hline & 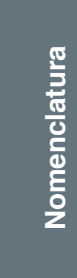 & & 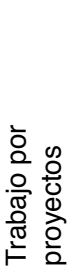 & 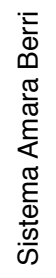 & 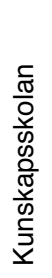 & 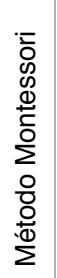 & 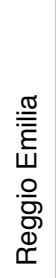 & 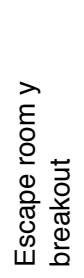 & 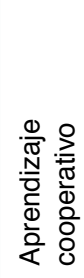 & 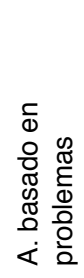 & 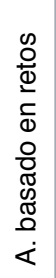 & 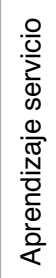 & 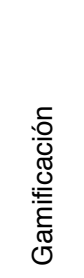 & 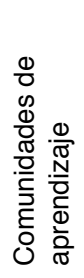 & 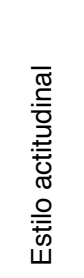 & 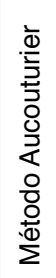 & 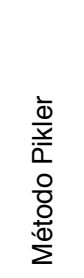 & 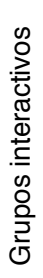 \\
\hline & $\dot{z}$ & & - & $N$ & $m$ & $\nabla$ & 10 & 0 & $\Lambda$ & $\infty$ & $\sigma$ & 으 & $\mp$ & $\cong$ & $\stackrel{m}{q}$ & $\underset{\sim}{+}$ & $\stackrel{2}{\circ}$ & $\stackrel{0}{\circ}$ \\
\hline
\end{tabular}




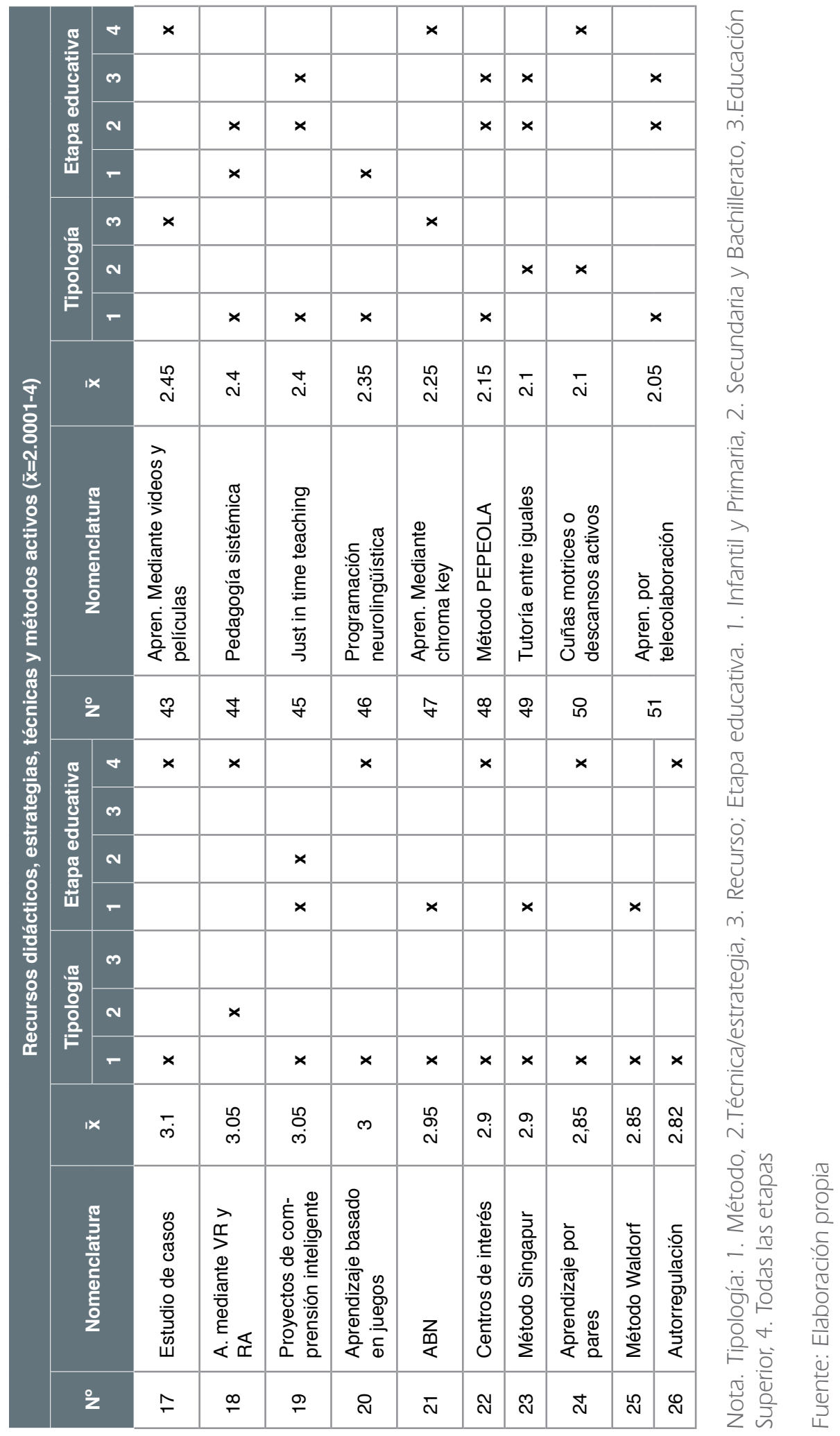




\section{Discusión y conclusiones}

En el estudio se han valorado y clasificado un total de 76 recursos, técnicas, estrategias y métodos didácticos, los cuales, han sido seleccionados tras llevar a cabo un proceso de revisión integradora en las bases de datos explicitadas. En este sentido, es necesario mencionar que no se ha encontrado en la literatura ninguna taxonomía donde se clasifiquen diferentes elementos de forma ecléctica en función de su carácter activo e instructivo y en función de la etapa o etapas educativas a las que mejor se adecúan en términos de utilización. En este sentido, el grueso de las mismas, se centran en una etapa o disciplina concreta. Un claro ejemplo de lo mencionado, es la clasificación establecida por Delgado (1991) en el ámbito de la Educación Física. En la misma, se establecen 6 grupos de clasificación de los estilos de enseñanza: (1) tradicionales, (2) individuales, (3) participativos, (4) socializadores, (5) cognitivos y (6) creativos; en los que se integran diferentes estilos de enseñanza en función de criterios como la actitud que adopta el profesorado, la dirección y organización de la clase, el control del docente y el contenido y la planificación del estudio entre otros. Es necesario mencionar que, en la presente clasificación, la taxonomía es realizada únicamente con el análisis establecido por el autor.

En el mismo ámbito de la Educación Física y con un carácter más reciente, es reseñable la clasificación de modelos pedagógicos, en la que se establecen, de forma similar a la presente taxonomía, 2 grandes grupos de clasificación para integrar los diferentes modelos pedagógicos -modelos pedagógicos consolidados y modelos emergentes- (Fernández-Rio et al., 2018).

Por otro lado, es destacable la clasificación de enfoques metodológicos universitarios elaborado por Alcoba (2012) y por Bourner (1997), en la que, de forma similar a nuestro estudio, tras una revisión bibliográfica y un procedimiento de panel de expertos, se establecieron un total de 22 y 52 enfoques metodológicos principales respectivamente, aunque estos no se clasificaron en categorías.

De forma paralela al caso anterior, Navaridas (2004), establece, 4 grupos de clasificación de técnicas, estrategias, métodos, modelos y estilos de enseñanza: (1) métodos tradicionales, (2) métodos cognitivos-conductuales, (3) métodos metacognitivos y (4) métodos de apoyo.

Por su parte, las clasificaciones establecidas por Borko et al. (2010) y OCDE (2016), quizás sean las que manifiestan más similitudes con esta investigación. La primera establece 2 grupos de clasificación de los métodos docentes: (1) enfoques tradicionales y (2) enfoques modernos o contemporáneos; y la segunda clasifica diversos métodos didácticos en función de las categorías aprendizaje dirigido, aprendizaje activo y activación cognitiva.

También existen numerosas investigaciones empíricas en las que se utilizan como variable dependiente diversos enfoques metodológicos, donde los criterios de inclusión de los mismos, no aparecen delimitados de forma exhaustiva, aunque, aun así, han servido para establecer la presente taxonomía (Crisol, 2012; Rodríguez-García \& Arias-Gago, 2019). También existen otras vinculadas a construcciones y revisiones teóricas que también se han considerado (De Miguel, 2009; Nieto \& Alfageme-González, 2017; Paños, 2017; Prieto et al., 2014; Silva \& Maturana, 2017; Toro \& Arguis, 2015).

A modo de conclusión, los resultados establecidos, materializados en una taxonomía elaborada desde un plano empírico a través de un grupo de expertos instruido ex profeso y al que se le administró una escala elaborada $a d$ hoc, han permitido clasificar 76 recursos, estrategias, técnicas y métodos didácticos de forma ecléctica en función del carácter instructivo o activo de cada uno y en función de la etapa educativa a la que mejor se adecúan y adaptan en términos de 
utilización (Tablas 4 y 5). Por tanto, esta taxonomía - la única en la literatura que considera los criterios citados- puede arrojar luz a los docentes a la hora de conocer y decidir qué métodos son más adecuados implementar en función de la etapa educativa, del papel que quiera adoptar en sus procesos de enseñanza y del papel que quiera otorgar a su alumnado en sus procesos de aprendizaje - activo o instructivo- Todas estas implicaciones adquieren una mayor relevancia, si cabe, debido a la complejidad terminológica de este ámbito de la didáctica y al carácter críptico de los diferentes conceptos, los cuales, han tratado de clarificarse en la introducción para facilitar la comprensión y permitir el avance en este aspecto esencial de la función docente.

A pesar de estas implicaciones, la investigación dispone de una serie de limitaciones: La primera, se relaciona con que en la presente publicación no se pudo detallar en profundidad la revisión establecida para seleccionar los 76 elementos metodológicos de la taxonomía. En este sentido, la resolución de la limitación citada abre una nueva línea de investigación centrada en la elaboración de una publicación donde se detalle la revisión implementada. La segunda limitación, se relaciona con la escasa delimitación conceptual de cada uno de los elementos que componen la taxonomía, esta circunstancia deja la puerta abierta a otra posible línea de investigación donde, por una parte, se defina en profundidad cada elemento metodológico $y$, por otra, a cada método se le asocien las estrategias y técnicas vinculadas. La última limitación, se vincula con la escasez de literatura nacional e internacional asociada a las taxonomías sobre aspectos metodológicos. Esta circunstancia otorga valor añadido a la investigación y ofrece a los docentes un referente a la hora de decidir qué recursos, estrategias, técnicas y métodos didácticos utilizar en función del papel que quiera dotar a su alumnado y la etapa educativa en la que se ubique el mismo.

\section{Referencias bibliográficas}

Alcoba, J. (2010). Los métodos de enseñanza en la estrategia docente de las Instituciones de Educación Superior. Un estudio sobre Escuelas de Negocios [Tesis Doctoral. Universidad Pablo de Olavide]. https://bit.ly/2ATkxmc

Alcoba, J. (2012). La clasificación de los métodos de enseñanza en Educación Superior. Contextos educativos, 15, 93-106. https://bit.ly/2ERU4nu

Andreu-Andrés, M.A., \& Labrador-Piquer, M.J. (2011). Formación del profesorado en metodologías y evaluación. Análisis cualitativo. Revista de investigación en Educación, 9(2), 236-245. https://bit.ly/2PRRcgM

Blackshields, D., Cronin, J.G.R., Higgs, B., Kilcommins, S., McCarthy, A., \& Ryan, A. (2016). Aprendizaje integrado. Investigaciones internacionales y casos prácticos. Narcea.

Blanchard, M., \& Muzás, M.D. (2016). Los proyectos de aprendizaje. Narcea.

Borko, H., Jacobs, J., \& Koellner, K. (2010). Contemporary approaches to teacher professional development. En P. Peterson, E. Baker y B. McGaw (Eds.), International Encyclopedia of Educaction (pp. 548-556). Elsevier. https://bit.ly/2St6Xg0

Bourner, T. (1997). Teaching methods for learning outcomes. Education + training, 39(9), 344348. https://bit.ly/2snL2Mz

Cantón, I., \& Pino, M. R. (2014). Organización de centros educativos en la sociedad del conocimiento. Alianza.

Castañeda M.B., Cabrera, A.F., Navarro, Y., \& De Vries, W. (2010). Procesamiento de datos y análisis estadísticos utilizando SPSS. Edipucrs

Colás, M.P., Buendía, L., \& Hernández, F. (2009). Competencias científicas para la realización de una tesis doctoral. Davinci.

Crisol, E. (2012). Opinión y percepción del profesorado y de los estudiantes sobre el uso de metodologías activas en la universidad de Granada [Tesis Doctoral, Universidad de Granada]. https://bit.ly/2UmXV45

De Miguel, M. (2009). Metodologías de enseñanza y aprendizaje para el desarrollo de competencias: Orientaciones para el profesorado univer- 
sitario ante el espacio europeo de Educación Superior. Alianza.

Delgado, M. A. (1991). Los estilos de enseñanza en la Educación Física. Servicio de publicaciones de la Universidad de Granada.

Díaz-Lucea, J. (1996). Los recursos y materiales didácticos en Educación Física. Apunts. Educación Física y Deportes, 1(43), 42-52. https://bit.ly/2zYxScG

Educacyl (2019). Fichas resumen de metodologías activas. https://bit.ly/2s9lsLg

Fernández-Balboa, J. M. (2003). La Educación Física desde una perspectiva crítica: De la pedagogía venenosa y el currículum oculto hacia la Dignidad. En V. López, R. Monjas, \& A. Fraile (Eds.), Los últimos diez años de la Educación Física Escolar. Universidad de Valladolid.

Fernández-March, A. (2006). Metodologías activas para la formación de competencias. Educatio Siglo XXI, 24, 35-56. https://bit.ly/39iKB6H

Fernández-Rio, J., Hortigüela, D., \& Pérez-Pueyo, A. (2018). Modelos pedagógicos en Educación Física. Ideas clave para incorporarlos al aula. Revista Española de Educación Física y deportes, 423, 57-80. https://bit.ly/37bpW2D

Gil, J. (2014). Metodologías didácticas empleadas en las clases de ciencias y su contribución a la explicación del rendimiento. Revista de Educación, 366 ,190-214.

http://dx.doi.org/10.4438/1988-592X-RE-2014-366-271

Guirao, S.J. (2015). Utilidad y tipos de revisión de literatura. ENE, Revista de Enfermería, 9(2). https://bit.ly/2ETzzqv

Hernández, C.A., \& Guárate, A.Y. (2017). Modelos didácticos para situaciones y contextos de aprendizaje. Narcea.

López-Pastor, V. M. (2009). La evaluación formativa y compartida en Educación Superior: Propuestas, técnicas, instrumentos y experiencias. Narcea.

Luelmo, M.J. (2018). Origen y desarrollo de las metodologías activas dentro del sistema educativo español. Encuentro, 27, 4-21. https://bit.ly/2MqfA7b

Marina, J.A., Pellicer, C., \& Manso, J. (2015). Libro Blanco de la profesión docente y su entorno escolar. Servicio de publicaciones del INTEF. https://bit.ly/3dw9ihR
Navaridas, F. (2004). Estrategias didácticas en el aula universitaria. Servicio de publicaciones de la Universidad de La Rioja. https://bit.ly/2PU0wkw

Nieto, J.M., \& Alfageme-González, M.B. (2017). Enfoques, metodologías y actividades de formación docente. Profesorado. Revista de currículum y formación del profesorado, 21(3), 63-81. https://bit.ly/2MqjuNk

OCDE (2016). Teaching strategies for instructional quality. Insights from the TALIS-PISA Data. OCDE publishing. https://bit.ly/2yzHHg9

Palma, M., Lagos, N., \& Donoso, M. (2017). Metodologías activas para la formación universitaria. Universidad del Bío-Bío. http://bit.ly/2BA7uTi

Palomares, A. (2011). El modelo docente universitario y el uso de nuevas metodologías en la enseñanza, aprendizaje y evaluación. Revista de Educación, 355, 591-604. https://bit.ly/2Nv5ulA

Paños, J. (2017). Educación emprendedora y metodologías activas para su fomento. Revista electrónica interuniversitaria de formación del profesorado, 20(3), 33-48.

http://dx.doi.org/10.6018/reifop.20.3.272221

Pérez-Pueyo, A., \& Hortigüela, D. (2020). ¿Y si toda la innovación educativa en Educación Física no es positiva en Educación Física? Reflexiones y consideraciones prácticas. Retos, 37, 579-587. https://bit.ly/2SpaE6u

Prieto, A., Díaz, D., \& Santiago, R. (2014). Metodologías inductivas. Digital text.

Rodríguez-García, A., \& Arias-Gago, A.R. (2019). Uso de metodologías activas. Un estudio comparativo entre profesores y maestros. Brazilian Journal of Development, 5(6), 5098-5111. https://bit.ly/2Zkuykk

Silva, J., \& Maturana, D. (2017). Una propuesta de modelo para introducir metodologías activas en Educación superior. Innovación educativa, 17(73), 117-131. https://bit.ly/2PZbXra

Tejedor, F.J., \& García-Valcárcel, A. (2012). Sociedad tecnológica e investigación educativa. Revista Española de pedagogía, 60(251), 3-26. https://bit.ly/2EW8xz6

Toro, A., \& Arguis, M. (2015). Metodologías activas. A tres bandas, (38), 69-77. https://bit.ly/34UzjSZ 
Tourón, J., \& Santiago, R. (2015). El modelo Flipped Learning y el desarrollo del talento en la escuela. Revista de Educación, 368, 196-223. https://10.4438/1988-592X-RE-2015-368-288

Vallejo, M., \& Molina, J. (2011). Análisis de las metodologías activas en el grado de maestro en educación infantil: la perspectiva del alumnado. REIFOP, 14(1), 207-217.
https://bit.ly/3dxVR0Z

Zabalza, M. A. (2003). Competencias docentes del profesorado universitario. Narcea.

Zemelman, S., Daniels, H., \& Hyde, A. (2005). Best practice: Today's standards for teaching and learning in America's schools. Heinemann. 